\title{
Discovery of Rigidified $\alpha, \beta$-Unsaturated Imines as New Resistance-breaking Insecticides for Malaria Vector Control
}

\author{
Alexander Arlt, Niels Böhnke, Sebastian Horstmann, Arnoldus W. P. Vermeer, Stefan Werner, \\ and Robert Velten*
}

\begin{abstract}
During our continuous search for new resistance-breaking insecticides applicable to malaria vector control, a new class of $\alpha, \beta$-unsaturated imines was identified by applying the principle of conformational rigidification as a powerful tool for compound optimisation. Herein we describe the successful synthesis of these compounds and their biological test results. Our lead compound $\mathbf{1 6}$ from this insecticidal class outperforms market standards, notably for the control of mosquito strains that exhibit either metabolic or target-site resistance to these established insecticides. In our model system for insecticide-treated mosquito nets the compound reveals long-lasting efficacy for up to several months.
\end{abstract}

Keywords: Anopheles · Conformational rigidification · Malaria $\cdot \alpha, \beta$-Unsaturated imines $\cdot$ Vinyl sulphides

\section{Introduction}

No new class of insecticides for control of adult malaria-vector mosquitoes has been approved by WHO for more than 30 years. Currently only two modes of action are in use, - i.e. sodium channel modulators (pyrethroids \& DDT) and acetylcholinesterase inhibitors (carbamates and organophosphates) - far fewer than are deployed in the agricultural sector. Therefore, metabolic and target-site resistance have evolved and become a major problem. New public health insecticides with activity against resistant strains are thus urgently needed. Since 2009, IVCC and Bayer have built up a strong partnership to discover such novel insecticides. During the last seven years, we have carefully investigated over 20 chemical classes, one of which will be presented in this paper.

\section{Discovery of Rigidified $\alpha, \beta$-Unsaturated Imines as New Insecticides}

In 2009 , a screening cascade to identify new insecticidal hits for the control of adult mosquitoes was established at Bayer. It was based on a high-throughput screening approach in which more than one million compounds from the Bayer substance library were tested against mosquito larvae. During the hit assessment compounds with an unselective mode of action (e.g. uncouplers of oxidative phosphorylation) were rejected. In order to identify resistance-breaking insecticides, the effect on adult mosquitoes with metabolic as well as target-site resistance to current mosquito insecticides was tested at an early stage in the screening cascade.

In the course of our screening pro- gramme, the $\beta$-arylthio- $\alpha, \beta$-unsaturated imine 1 was identified as an interesting starting point for chemical optimisation. Researchers at Sumitomo had originally discovered such compounds: in 2007 and 2009, Itoh et al. described the insecticidal effects of thioimidates (formally thioethers of imines) such as $\mathbf{2}$ and $\mathbf{3}$, as well as of imines like 1 (Fig. 1).[1,2] Our strategy was to improve the potency of the highly conformationally-flexible imine $\mathbf{1}$ by constraining the molecule in its bioactive conformation. Therefore, we designed analogues of the $\alpha, \beta$-unsaturated imine $\mathbf{1}$ that were rigidified by an additional ethylene bridge, i.e. the tetralin-type derivative 5 (Fig. 1). This approach differs from work conducted at Nippon Soda, which was focussed on the insecticidal activity of cyclic thioimidates such as $4 .{ }^{[3]}$ Rigidified $\alpha, \beta$ unsaturated imines such as $\mathbf{5}$ were novel

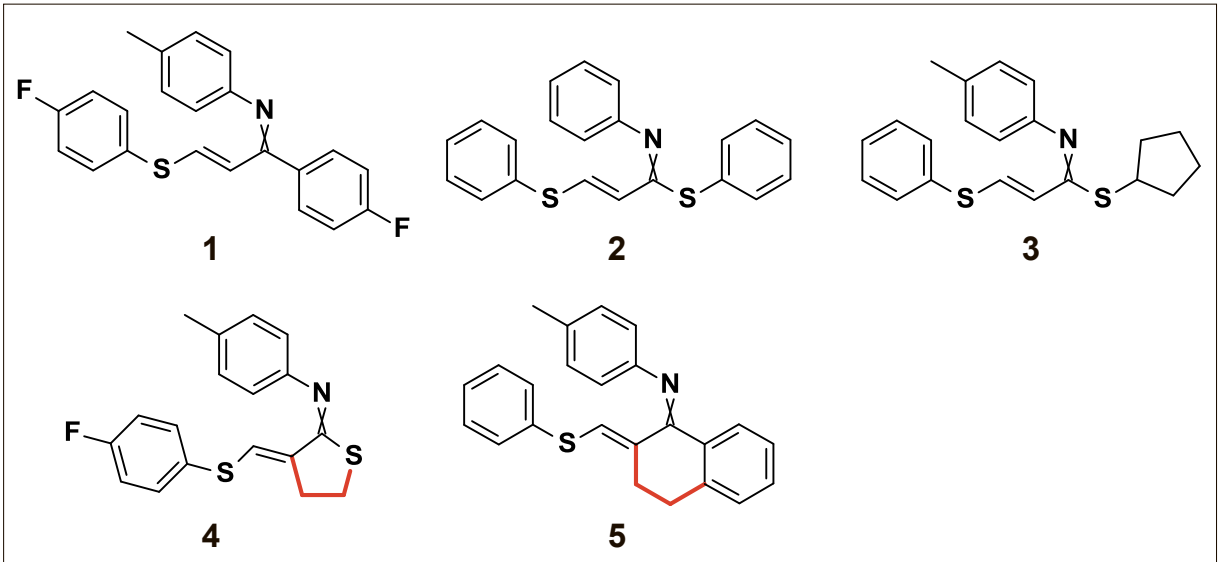

Fig. 1. Structures of insecticidal $\alpha, \beta$-unsaturated thioimidates and imines. Rigidification is highlighted in red. 
and their synthesis and biological efficacy were first described by Bayer in 2012. [4]

A first retrosynthetic analysis of the $\beta$-arylthio- $\alpha, \beta$-unsaturated imines might suggest that ketones such as $\mathbf{6}$ could serve as an appropriate synthetic precursor. However, it is well known that these compounds react preferentially with anilines at the $\beta$-position resulting in enaminones like 7 (Scheme 1). ${ }^{[5]}$ Our synthetic strategy therefore had to rely on the generation of the vinyl sulphide from a thioacetal after the formation of the imine. For example, the required thioacetal $\mathbf{1 0}$ was easily prepared from silyl enol ether $\mathbf{9}$ and (phenylthio)orthoformate as starting materials using a method described by Trost et al. ${ }^{[6]}$ (Scheme 1). The formation of the imine $\mathbf{1 1}$ was then successfully mediated by titanium tetrachloride. The use of substoichiometric amounts of titanium tetrachloride, quenching with triethylamine or DBU and a thorough purification was essential in this step to prevent the formation of enamine 8. In the final step, a catalytic amount of titanium tetrachloride was sufficient to effect the elimination of thiophenol to provide the final product 5. ${ }^{[4]}$

The rigidified $\alpha, \beta$-unsaturated imine $\mathbf{5}$ showed surprisingly good efficacy against a resistant strain $(k d r)$ of Anopheles gambiae ${ }^{[7]}$ with $0.8 \mathrm{mg} / \mathrm{m}^{2}$ as the lowest applied dosage which provided $80 \%$ efficacy ${ }^{[8]}$ (Table 1) and was our first lead compound within this chemical class.

More than 100 analogues of the first lead structure $\mathbf{5}$ were prepared following the methodology described above (Scheme 1). ${ }^{[4]}$ In the first cycle of optimisation the application of 'fluorine and methyl walks'

Table 1. Laboratory efficacies of rigidified $\alpha, \beta$-unsaturated imines and insecticidal standards against a homozygous resistant strain (kdr) of Anopheles gambiae.

\begin{tabular}{|c|c|}
\hline Compound & Efficacy ${ }^{\mathrm{a}}, \mathrm{mg} / \mathrm{m}^{2}$ \\
\hline $\mathbf{1}$ (Sumitomo) & 0.8 \\
\hline $\mathbf{4}$ (Nippon Soda) & 4 \\
\hline $\mathbf{5}$ & 0.8 \\
\hline $\mathbf{1 2}$ & 0.16 \\
\hline $\mathbf{1 3}$ & 0.16 \\
\hline $\mathbf{1 4}$ & $>100$ \\
\hline $\mathbf{1 5}$ & $>100$ \\
\hline $\mathbf{1 6}$ & 0.032 \\
\hline $\mathbf{1 7}$ & 0.16 \\
\hline Bendiocarb & 0.8 \\
\hline DDT & $>100$ \\
\hline Permethrin & 4 \\
\hline Deltamethrin & 0.16 \\
\hline
\end{tabular}

aLowest applied dosage which provides > $80 \%$ mortality after 24 h (30 min exposure).

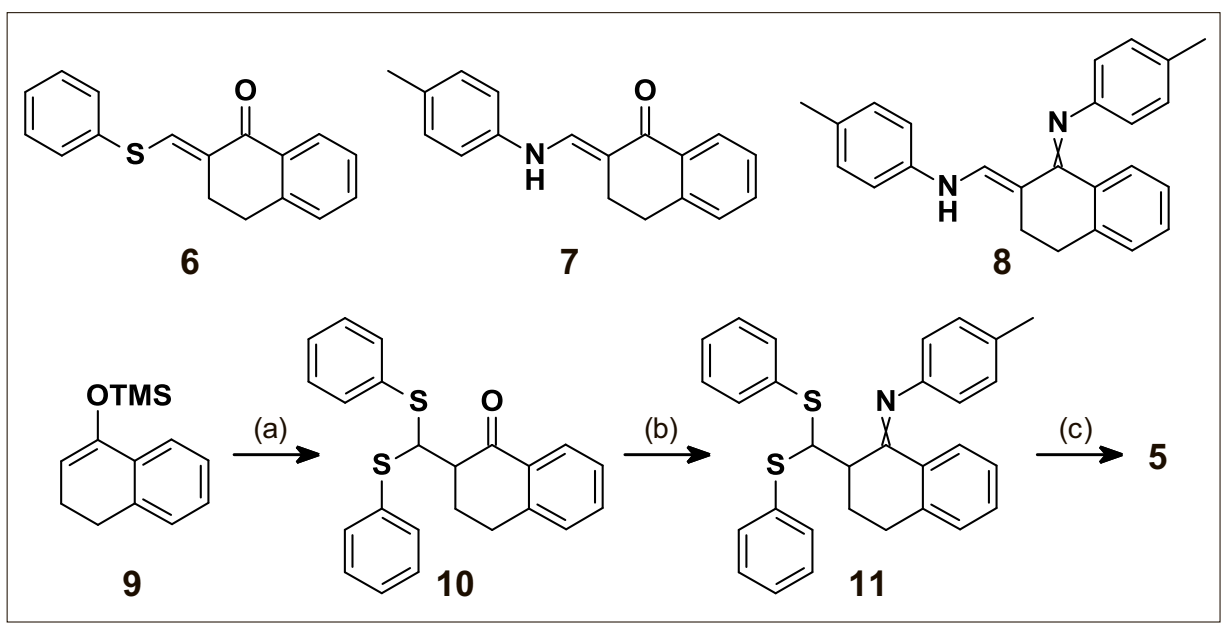

Scheme 1. Synthesis of rigidified $\alpha, \beta$-unsaturated imine 5 using a thioacetal. Reagents and conditions: (a) $(\mathrm{PhS})_{3} \mathrm{CH}, \mathrm{SnCl}_{4}, \mathrm{CH}_{2} \mathrm{Cl}_{2}$; (b) $\mathrm{ArNH}_{2}, 0.5$ eq TiCl 4 , toluene; (c) 0.3 eq TiCl , toluene. ${ }^{[4]}$ allowed us to identify several aromatic substitution positions in which fluorine atoms or methyl groups were tolerated or even improved insecticidal activity, for example compounds 12 and 13 (Fig. 2). In contrast, more bulky groups such as isopropyl or phenyl resulted in a significant drop of the insecticidal activity as displayed by analogues 14 and 15, respectively. A subsequent optimisation campaign with the optimal fluorine or methyl substituent in place, and variation of the ethylene bridge led to the discovery of very potent chromane-type and ring-contracted indanetype analogues, such as compounds $\mathbf{1 6}$ and 17, respectively (Fig. 2).
The structure of our lead compound $\mathbf{1 6}$ was confirmed by a single crystal X-ray diffraction study (Fig. 3). It shows that - at least in the solid state - the configuration of the carbon-nitrogen double bond is $\mathrm{Z}$. The $N$-aryl group is twisted, sacrificing the planarity of a fully conjugated system.

\section{Biological Properties of Lead Compound 16}

Table 2 shows the activity of our lead compound $\mathbf{1 6}$ against a set of susceptible and resistant Anopheles strains in a comparison with four important market stan-

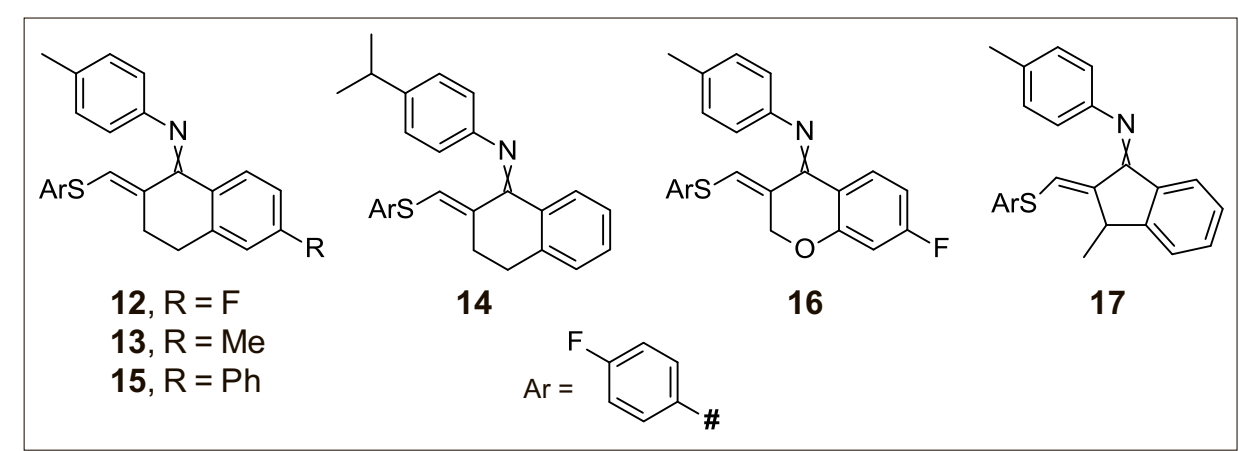

Fig. 2. Structures of some rigidified $\alpha, \beta$-unsaturated imines.

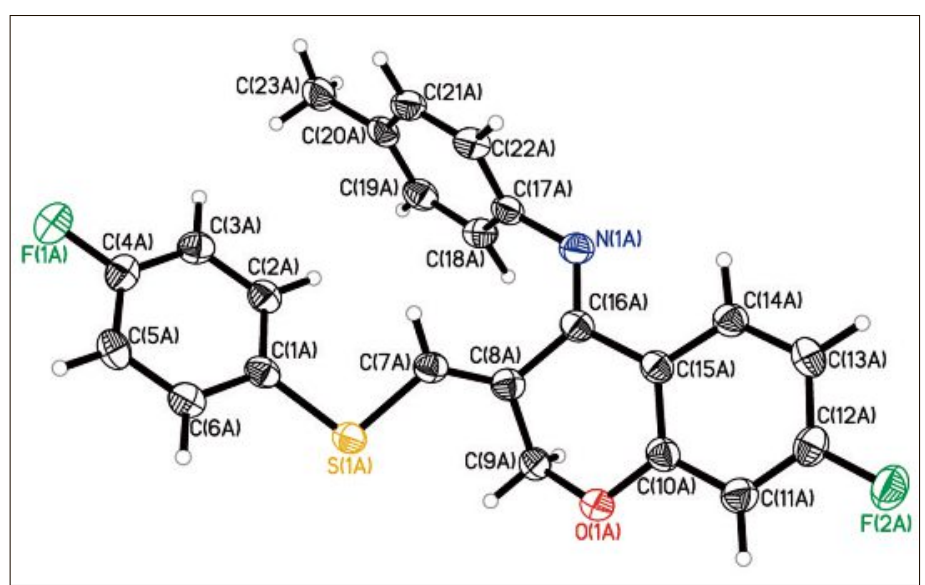

Fig. 3. Single crystal X-ray structure of rigidified $\alpha, \beta-$ unsaturated imine 16. 
Table 2. Laboratory efficacies ${ }^{a}$ of market standards and compound $\mathbf{1 6}$ against a broad range of susceptible and resistant Anopheles strains. Resistance factors are given in brackets. ${ }^{b}$

\begin{tabular}{|c|c|c|c|c|c|}
\hline Strain & Bendiocarb & DDT & Permethrin & Deltamethrin & 16 \\
\hline An. gambiae, Kisumu ${ }^{\mathrm{c}}$ & 0.8 & 0.8 & 0.16 & 0.0064 & 0.16 \\
\hline An. gambiae, $\mathrm{RDL}^{\mathrm{d}}$ & $0.8(1)$ & $0.8(1)$ & $0.16(1)$ & $0.0064(1)$ & $0.16(1)$ \\
\hline An. gambiae, Akron ${ }^{\mathrm{e}, \mathrm{f}}$ & $>100(>125)$ & not tested & $0.8(5)$ & $0.0064(1)$ & $0.8(5)$ \\
\hline An. gambiae, $\mathrm{RSPH}^{\mathrm{g}}$ & $0.8(1)$ & $>100(>125)$ & $4(25)$ & $0.16(25)$ & $0.032(0.2)$ \\
\hline An. gambiae, Tiassaléf,h & $20(25)$ & $>100(>125)$ & $100(625)$ & $4(625)$ & $0.16(1)$ \\
\hline An. funestus, FUMOZ-R ${ }^{\mathrm{i}}$ & $0.8(1)$ & $0.16(0.2)$ & $20(125)$ & $20(3125)$ & $0.8(5)$ \\
\hline
\end{tabular}

aLowest applied dosage in $\mathrm{mg} / \mathrm{m}^{2}$ which provides $>80 \%$ mortality after $24 \mathrm{~h}$ (30 min exposure); ${ }^{\mathrm{b}}$ Resistance factor (RF) = efficacy resistant strain / efficacy susceptible strain (An. gambiae, Kisumu); 'Susceptible strain, Malaria Research and Reference Reagent Resource Center (MR4);

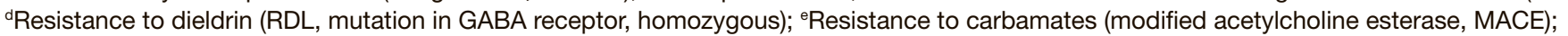
${ }^{\dagger}$ Mosquitoes were kindly provided by the Liverpool Insect Testing Establishment (LITE); ${ }^{9}$ Resistance to pyrethroids (East African kdr mutation in sodium channel, homozygous) and elevated levels of glutathione S-transferases (GSTs, metabolism of DDT); hWest African kdr mutation in sodium channel, MACE, and elevated levels of cytochrome P450 monooxygenases (metabolism of pyrethroids); 'Elevated levels of cytochrome P450 monooxygenases (metabolism of pyrethroids).

dards (bendiocarb, DDT, permethrin, and deltamethrin). Resistant strains from Bayer and from the Liverpool Insect Testing Establishment (LITE) were used to represent both target-site $(k d r, \mathrm{RDL}, \mathrm{MACE})$ and metabolic resistance mechanisms (elevated levels of cytochrome P450 monooxygenases or glutathione $S$-transferases).

Whilst the pyrethroids deltamethrin and permethrin lacked activity against the metabolically resistant Anopheles gambiae Tiassalé and An. funestus FUMOZ-R strains (resistance factors RF $>>100$ ) in our laboratory screening assay, ${ }^{[8]}$ bendiocarb activity was especially affected by strains with an acetylcholine esterase target-site mutation (e.g. in An. gambiae Akron, RF > 125). However the sodium channel target-site mutation $k d r$ (knockdown resistance, e.g. in An. gambiae $\mathrm{RSPH})$ resulted in only a moderate decrease in pyrethroid activity, with a RF of 25 . In contrast to the performance of these market standards, the new $\alpha, \beta$-unsaturated imine 16 showed good control of all resistant strains even at $0.8 \mathrm{mg} / \mathrm{m}^{2}$. No significant cross-resistance was observed $(\mathrm{RF}<10)$. This led us to assume that this compound has a mode of action that is different from those of existing insecticides used for malaria vector control.

Long-lasting insecticide-treated mosquito nets (LLINs) and indoor residual spraying (IRS) are the main techniques for malaria vector control. LLINs are nets treated in the factory with an insecticide that is either incorporated into the net fabric, or coated on it. To meet WHO guidelines an LLIN must retain its biological activity after at least 20 washes and three years of use under field conditions. ${ }^{[9]}$ The residual efficacy of an $\alpha, \beta$-unsaturated imine such as $\mathbf{1 6}$ on a glazed tile is very disappointing unless it is applied in a suitable formulation. The activity of the unformulated active ingredient $\mathbf{1 6}$ clearly decreased by a factor of more than one hundred within two weeks. However, when protected from environmental influences by incorporation in polyethylene micro granules (approximate size $200 \mu \mathrm{M}$, Fig. 4), as a suitable model system for insecticide-treated bed nets, this new insecticide shows at a $0.5 \%$ w/w concentration a strong and undiminished control of mosquitoes over a sixmonth testing period.

Although there was no evidence of any acute oral mammalian toxicity at doses of up to $300 \mathrm{mg} / \mathrm{kg}$ body weight in rats, we observed that all of our best compounds showed severe cytotoxicity and genotoxicity in vitro. An explanation for the latter behaviour might be the release of anilines from the compound through hydrolysis or metabolic degradation. A diverse set of analogues of $\alpha, \beta$-unsaturated imine $\mathbf{1 6}$ was prepared spanning a range of electronrich and electron-poor anilines. Ultimately, however, we were unable to significantly lower the genotoxicity whilst retaining strong insecticidal activity.
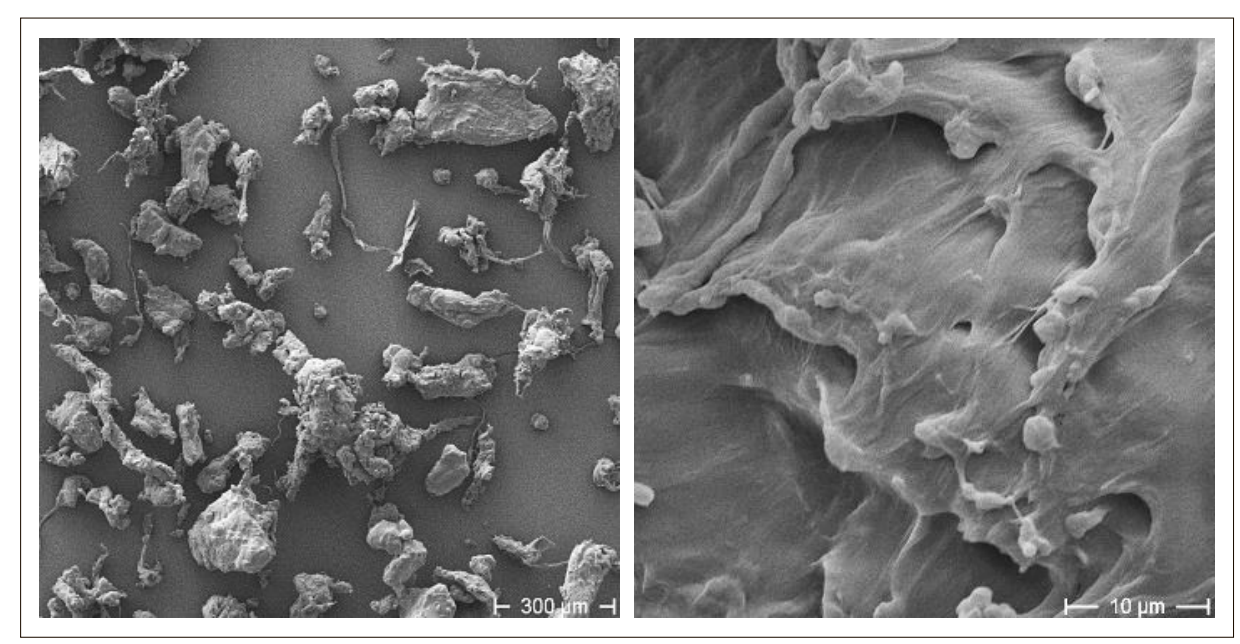

Fig. 4. Scanning electron microscope images of polyethylene micro granules (size of roughly

$200 \mu \mathrm{M})$ as suitable model system for insecticide treated bed nets.

\section{Conclusions}

The rigidified $\alpha, \beta$-unsaturated imines are a novel class of insecticides that are able to control adult malaria-vector mosquitoes. The best compound $\mathbf{1 6}$ outperforms the market standards with respect to the control of important metabolic and/or target-site resistant mosquito strains. In our model system for long-lasting insecticidetreated mosquito nets it showed sustained strong efficacy, even after several months. Therefore it fulfilled several of the key criteria for a new malaria vector control product. However, ultimately we were unable to overcome critical toxicology issues with this chemical class.

\section{Acknowledgements}

The authors are indebted to Carmen Dunkel, Anja Hanke, Roman Hanke, Julia Hiedels, Klaus-Dieter Huth, Valentina Neufert, and Holger Rings for synthesis of many of the compounds, to Rolf Jung, Katja Falke and Kristin Raschke for performing the con- 
tact bioassays on different insects, Dr. Ulrich Ebbinghaus-Kintscher for the biochemical studies, Dr. Helen Tinwell for toxicological studies, Emilia Hilz for the formulation work, and to Drs. Arnd Voerste and Kerstin Ilg for their managerial leadership. Furthermore, we are grateful to the Liverpool Insect Testing Establishment, which helped us to test several resistant mosquito strains, and to Prof. Dr. Maureen Coetzee from the South African National Institute for Communicable Diseases for providing the An. funestus FUMOZ-R strain and especially for her valuable support in the establishment of this strain at Bayer. Last but not least, all of us would like to personally thank our colleagues from the IVCC, especially Prof. Dr. Trevor Perrior, Dr. Colin Ruscoe, and Dr. Robert Sloss for numerous fruitful discussions and a marvellous collaboration over the past few years.

Received: June 29, 2016
[1] S. Itoh, A. Iwata, WO Patent Appl. No. WO2007063702, 2007.

[2] S. Itoh, T. Kuragano, WO Patent Appl. No. WO2009048152, 2009.

[3] K. Kutose, J. Takahashi, I. Hamamoto, M. Yano, J. Morohoshi, J. Kanazawa, WO Patent Appl. No. WO2010070910, 2010.

[4] S. Werner, U. Ebbinghaus-Kintscher, S Horstmann, M. Maue, H.-G. Schwarz, R. Velten, A. Voerste, U. Goergens, A. Turberg, WO Patent Appl. No. WO2012072489, 2012.

[5] T. Nishio, Y. Omote, Synthesis 1980, 390.

[6] B. M. Trost, P. Seoane, S. Mignani, M. Acemoglu, J. Am. Chem. Soc. 1989, 111, 7487.

[7] Resistance to pyrethroids (East African $k d r$ mutation in sodium channel) and elevated levels of glutathione $S$-transferases (GSTs, metabolism of DDT)
[8] Lowest applied dosage in $\mathrm{mg} / \mathrm{m}^{2}$ which provides $>80 \%$ mortality after $24 \mathrm{~h}$. The compounds were tested in a contact bioassay on glazed tiles. In order to treat the tile with active ingredient, technical grade active ingredient was dissolved in acetone and this mixture evenly distributed onto a glazed tile. After evaporation of the acetone the test mosquitoes were placed onto the tile and exposed to the active ingredient for 30 minutes. After the exposure time the mosquitoes were transferred to clean storage receptacles and checked for mortality in comparison to control insects that had contacted a tile treated with acetone only.

[9] World Health Organization, guidelines for laboratory and field testing of longlasting insecticidal nets (WHO/HTM/NTD/ WHOPES/2013.1) 